\title{
First Principles Calculations of Optical Absorption Spectra of Atoms in the Vacuum and Crystals
}

\author{
Kaoru Ohno ${ }^{a, *}$, Miou Furuya ${ }^{a}$, Soh Ishii ${ }^{a}$, \\ Yoshifumi Noguchi ${ }^{a}$, Shohei Iwata ${ }^{a}$, Yoshiyuki Kawazoe ${ }^{b}$, \\ Shinichiro Nagasaka ${ }^{c}$, Takehisa Yoshinari ${ }^{c}$ and \\ Yoshio Takahashi ${ }^{\mathrm{c}}$

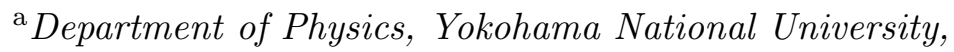 \\ 79-5 Tokiwadai, Hodogaya-ku, Yokohama 240-8501, Japan \\ ${ }^{\mathrm{b}}$ Institute for Materials Research, Tohoku University, \\ 2-1-1 Katahira, Aoba-ku, Sendai 980-8577, Japan \\ ${ }^{\mathrm{c}}$ Department of Physics, Yamagata University, \\ 1-4-12 Koshirakawacho, Yamagata, 990-8560, Japan
}

\begin{abstract}
Starting from the $G W$ approximation (GWA) beyond the density functional theory and taking into account the excitonic effect, we have determined optical properties of isolated $\mathrm{Zn}$ atom and also $\mathrm{Cu}$ impurity atom in $\mathrm{NaCl}$ crystal. In the representation of single electron wave functions, we have used the all-electron mixed basis approach in which both plane waves and atomic orbitals are used as a basis set. The resulting quasipartile energies and optical absorption spectra are compared with available experimental data.
\end{abstract}

Key words: first principles, quasiparticle energy, optical absorption spectra, many-body perturbation theory, Bethe-Salpeter equation

* Corresponding author. Tel.: +81-45-339-4254; Fax: +81-45-338-3020

Email address: ohno@ynu.ac.jp (Kaoru Ohno). 


\section{Introduction}

In small clusters, spectra of electronic excited states are strongly dependent on the size of the cluster, and the size control would become a useful technique in the frequency tuning of the excitation spectra of small clusters [1]. In particular, the optical absorption and the fluorescence may play an important role in the application to optical devises, for example.

In spite of these interests, the systematic theoretical study of the spectra of the quasiparticle energies in atomic scale systems in terms of the state-of-the-art $G W$ approximation (GWA) [2-4] for the electron self-energy (constructed by the one-particle Green's function $G$ and the dynamically screened Coulomb interaction $W$ on the basis of the many-body perturbation theory) has been very limited. Moreover, the desirable calculation of the optical absorption spectra of small clusters starting from the GWA and taking the excitonic effect into account for the two-particle Green's function [5-7] has been very limited also.

Previously some of the present authors have applied the GWA to the calculation of the quasiparticle energy spectra of small clusters such as $\mathrm{Li}_{n}, \mathrm{Na}_{n}$, $\mathrm{K}_{n}[8,9]$ and $\mathrm{Si}_{n}$ [10]. More recently, Ohno [11] presented a result of optical absorption spectra of alkali-earth atoms and small sodium clusters by starting from the GWA and solving the Bethe-Salpeter (BS) equation for the twoparticle Green's function which takes the excitonic effect into account. The method uses the all-electron mixed basis approach in which one-particle wave functions are expressed by both atomic orbitals and plane waves [7,12-14]. The results are fairly in good agreement with experiments.

In the present report, we will apply this approach to the investigation of the quasiparticle and optical absorption spectra of atoms in the vacuum and crystals. In particular, we treat transition metal elements. This became possible because we have recently made it possible to deal with the $d$ atomic orbitals in our all-electron mixed basis $G W$ code. Here, for the system of an isolated atom in the vacuum, we investigate zinc atom, while, for the system of an atomic impurities in crystals, we investigate $\mathrm{Cu}^{+}$ion embedded in the sodium-chloride $(\mathrm{NaCl})$ crystal.

For the calculation of $\mathrm{Cu}^{+}$ion embedded in the sodium-chloride crystal, we use $2 \times 2 \times 2$ simple cubic supercell and replace one sodium atom with a $\mathrm{Cu}$ atom. The position of the $\mathrm{Cu}$ atom has been already determined by our previous study using the first-principles structural optimization [15].

The rest of this paper is organized as follows. In section 2, we briefly describe the methodology. In section 3, we present the result of our calculation. We will summarize this paper in section 4 . 


\section{Methodology}

Quasiparticle energies of real atomic systems can be calculated accurately by means of the GWA [2-4] which treats the first-order contribution to the selfenergy of the one-particle Green's function $G$ with respect to the dynamically screened Coulomb interaction $W$ given in the random-phase approximation (RPA).

In the GWA, the quasiparticle energies $\varepsilon_{n}^{\mathrm{QP}}$ and wave functions $\psi_{n}(\mathbf{r})$ are obtained by solving

$$
\begin{aligned}
& \left(T+V_{e x t}+V_{H}\right) \psi_{n}(\mathbf{r})+\int d \mathbf{r}^{\prime} \Sigma\left(\mathbf{r}, \mathbf{r}^{\prime} ; \varepsilon_{n}^{\mathrm{QP}}\right) \psi_{n}\left(\mathbf{r}^{\prime}\right)=\varepsilon_{n}^{\mathrm{QP}} \psi_{n}(\mathbf{r}), \\
& \Sigma\left(\mathbf{r}, \mathbf{r}^{\prime} ; \omega\right)=\frac{i}{2 \pi} \int d \omega^{\prime} G\left(\mathbf{r}, \mathbf{r}^{\prime} ; \omega+\omega^{\prime}\right) W\left(\mathbf{r}, \mathbf{r}^{\prime} ; \omega^{\prime}\right) e^{i \eta \omega^{\prime}}
\end{aligned}
$$

where $T, V_{\text {ext }}, V_{H}$, and $\Sigma$ are the kinetic energy operator, external potential, Hartree potential, and electron self-energy containing the effect of exchangecorrelation between electrons, respectively; $\eta$ is a positive infinitesimal number. $W$ is the dynamically screened Coulomb interaction expressed symbolically as $W=\varepsilon^{-1} U$ in the random phase approximation (RPA), where $U$ is the bare Coulomb interaction and $\varepsilon$ is the dielectric function $\varepsilon=1-4 \pi P$ (and $P=-i G G$ is the polarizability). Here the generalized plasmon pole model (GPP) [3] is used to evaluate the self-energy. The one-particle Green's function $G$ is given by

$$
G\left(\mathbf{r}, \mathbf{r}^{\prime}, \omega\right)=\sum_{n^{\prime}} \frac{\psi_{n^{\prime}}(\mathbf{r}) \psi_{n^{\prime}}^{*}\left(\mathbf{r}^{\prime}\right)}{\omega-\varepsilon_{n^{\prime}}-i \delta_{n^{\prime}}}
$$

where $\delta_{n^{\prime}}=0^{+}$for $\varepsilon_{n^{\prime}}<\mu_{\mathrm{F}}$ and $\delta_{n^{\prime}}=0^{-}$for $\varepsilon_{n^{\prime}}>\mu_{\mathrm{F}}$, provided that $\mu_{\mathrm{F}}$ is the Fermi energy. Conventionally $\psi_{n^{\prime}}(\mathbf{r})$ and $\varepsilon_{n^{\prime}}^{\mathrm{QP}}$ have been replaced by the LDA wavefunctions and the LDA energy eigenvalues $\varepsilon_{n^{\prime}}^{\text {LDA }}$. Then, quasiparticle energies are obtained in terms of first-order perturbation theory as

$$
\varepsilon_{n}^{\mathrm{QP}} \approx \varepsilon_{n}^{\mathrm{LDA}}+\int d \mathbf{r} \int d \mathbf{r}^{\prime} \psi_{n}^{*}(\mathbf{r})\left[\Sigma\left(\mathbf{r}, \mathbf{r}^{\prime}, \varepsilon_{n}^{\mathrm{QP}}\right)-\mu_{\mathrm{xc}}^{\mathrm{LDA}}(\mathbf{r}) \delta\left(\mathbf{r}-\mathbf{r}^{\prime}\right)\right] \psi_{n}\left(\mathbf{r}^{\prime}\right)
$$

So far, many calculations with this GWA on the $a b$ initio level have been performed for variety of crystals and surfaces [7]. Recently, we have calculated quasiparticle energy spectra of small alkali-metal clusters [8,9] as well as small silicon clusters [10], by using our $G W$ code implemented on the all-electron 
mixed basis approach $[7-11,13,14]$, in which single particle wave functions are expanded with both plane waves (PW's) and atomic orbitals (AO's).

It has been recognized that spectroscopic properties involving two-particle excitations should be determined still beyond the GWA, i.e., by evaluating accurately the two-particle Green's function. The excitonic effect in the optical absorption spectra can be taken into account by summing the electron-hole ladder diagrams, which become important in particular at short distances, in the two-particle Green's function. This can be formulated by using the functional derivative of the electron self-energy $U^{\text {Hartree }}+\Sigma^{G W}$ with respect to the one-particle Green's function $G$. Thus derived equation is the BS equation for the two-particle Green's function, $S\left(1,1^{\prime} ; 2,2^{\prime}\right)=S\left(\boldsymbol{r}_{1}, t_{1}, \boldsymbol{r}_{1}^{\prime}, t_{1}^{\prime} ; \boldsymbol{r}_{2}, t_{2}, \boldsymbol{r}_{2}^{\prime}, t_{2}^{\prime}\right)$, $[7]$

$$
S\left(1,1^{\prime} ; 2,2^{\prime}\right)=S_{0}\left(1,1^{\prime} ; 2,2^{\prime}\right)+S_{0}\left(1,1^{\prime} ; 3,3^{\prime}\right) \Xi\left(3,3^{\prime} ; 4,4^{\prime}\right) S\left(4,4^{\prime} ; 2,2^{\prime}\right) .
$$

Here, $S_{0}\left(1,1^{\prime} ; 2,2^{\prime}\right)=G\left(1^{\prime}, 2^{\prime}\right) G(2,1)$ is the unperturbed function, in which the disconnected term $-G\left(1,1^{\prime}\right) G\left(2,2^{\prime}\right)$ is excluded. (The notation $G(1,2)=$ $G\left(\boldsymbol{r}_{1}, \boldsymbol{r}_{2} ; t_{1}-t_{2}\right)$ is used to express the one-particle Green's function.) The interaction kernel $\Xi$, which stands for the irreducible electron-hole interaction, is determined by $\Xi=\delta\left(U^{\text {Hartree }}+\Sigma^{G W}\right) / \delta G$ and contains two different contributions

$$
\Xi\left(1,1^{\prime} ; 2,2^{\prime}\right)=-i \delta\left(1,1^{\prime}\right) \delta\left(2,2^{\prime}\right) U(1,2)+i \delta(1,2) \delta\left(1^{\prime}, 2^{\prime}\right) W^{\prime}\left(1,1^{\prime}\right)
$$

where $U(1,2)=1 /\left|\boldsymbol{r}_{1}-\boldsymbol{r}_{2}\right|$ comes from the functional derivative of the Hartree term $\left(U^{\text {Hartree }}\right)$, and $-W^{\prime}\left(1,1^{\prime}\right)$ represents the screened Coulomb attraction coming from the functional derivative of the $G W$ self-energy $\left(\Sigma^{G W}\right)$.

To solve (6), one has to invert a four-point function $I-S_{0} \Xi$, where $I$ is the identity operator. Strinati [16] derived a simplified eigenvalue equation which can be used to bypass the matrix inversion of the BS equation. Strinati treated positive frequency part only. Albrecht et al. [5] derived and applied a similar eignevalue equation with static approximation (they treated negative frequency part as well). Rohlfing and Louie [6] applied the Strinati scheme combined with the generalized plasmon-pole model. All these authors have replaced the kernel $W^{\prime}$ with the dynamically screened interaction $W$, which is equivalent to neglecting the term $G \delta W / \delta G$ in $\delta \Sigma / \delta G$ [16]. For the contribution of this $G \delta W / \delta G$ as well as the negtive frequency part, an analysis is given by Ohno [11].

The basic equation stands for $[17]$

$$
\left(\varepsilon_{c}^{\mathrm{QP}}-\varepsilon_{v}^{\mathrm{QP}}\right) A_{v c}+\sum_{v^{\prime} c^{\prime}}\left\{2 X_{v c ; v^{\prime} c^{\prime}} \delta_{M, 0}-W_{v c ; v^{\prime} c^{\prime}}(\Omega)\right\} A_{v^{\prime} c^{\prime}}=\Omega A_{v c}
$$


In (7), the exchange term $X$ exists only for singlet exciton, having spin multiplicity $M=0$. Moreover, if only one diagonal element between $v=v^{\prime}=$ HOMO and $c=c^{\prime}=$ LUMO dominates the others, the magnitude of the singlet-triplet splitting in the optical absorption peaks amounts to $2 X_{v c ; v c}$, which is a well known result.

In the following calculation of Zn, a rhombohedoral fcc supercell is used with a cubic edge of 30 a.u.. As a basis functions, 2975 PW's corresponding 8.7 Ry cutoff energy as well as $1 s, 2 s, 2 p, 3 s, 3 p, 3 d$ and $4 s$ AO's are used. In the evaluation of matrix elements, $2975 \mathbf{G}\left(\mathbf{G}^{\prime}\right)$ vectors corresponding 8.7 Ry cutoff energy are used for $\Sigma_{c}(\omega)$ and $(W-U)$, and $40857 \mathbf{G}$ vectors corresponding 50 Ry cutoff energy are used for $\Sigma_{x}$ and $U$. In addition, integrations along the radial coordinate are used to evaluate matrix elements of $\Sigma_{x}$ and $U$ sandwiched by the on-site AO's only. In the calculation of $\mathrm{Cu}$ impurity in sodium-chloride crystal, $2 \times 2 \times 2$ simple-cubic unit cell is employed, and only $\Gamma$ point is used in place of doing the $k$-point sampling. According to our separate study on the optimal position of the $\mathrm{Cu}$ atom [15], we assume two possibilities for the position of the $\mathrm{Cu}$ atom: One is the on-center positoin and the other is the off-center position $7 \%$ deviated along the (111) axis, i.e. $(0.57,0.57,0.57)$. The local relaxation of the ligand crystal is also taken into account in the present calculation.

\section{Results}

First we present the result of $\mathrm{Zn}$ atom. Table 1 lists all the terms contributing the GW quasiparticle energies of the HOMO $(4 s)$ state and the LUMO $(4 p)$ states. The columns indicated as 'exact' represent the values computed directly with the Herman-Skillman atomic code, which can give exact LDA values for isolated atoms. The experimental value for the HOMO state is the ionization potential with a minus sign. An excellent agreement is achieved between the resulting $G W$ quasiparticle energy and the experimental value. The resulting optical absorption peak energy of isolated $\mathrm{Zn}$ atom is presented in Table 2 together with the experimental value. The energy depends on the character of the excited states, either the singlet $\left({ }^{1} S \rightarrow{ }^{1} P\right)$ exciton or the triplet $\left({ }^{1} S \rightarrow{ }^{3} P\right)$ exciton. Agreement between the present result and the experimental value is fairly good, but the singlet-triplet (exchange) splitting is slightly larger than the experimental value. This is a common feature of the present method $[11,17]$ and due to the fact that the amount of this exchange splitting is directly related to the exchange integral sandwitched by the LDA wave functions. Only the absolute values of singlet and triplet absorption energies reflect the details of the Bethe-Salpeter equation and their difference (i.e., the exchange splitting) does not directly. 
Table 1

Contributions to the quasiparticle energies (in eV) for the HOMO and LUMO states are shown for $\mathrm{Zn}$ atom compared with the experimental ionization potential and negative of the electron affinity $\left(-E^{\mathrm{EXP}}\right) . \mu_{\mathrm{x} c, n}^{\mathrm{LDA}}=\left\langle n\left|\mu_{\mathrm{x} c}^{\mathrm{L} D A}\right| n\right\rangle$ means the expectation value of the LDA exchange-correlation potential, $\Sigma_{\mathrm{x}, n}$ and $\Sigma_{\mathrm{c}, n}$ are, respectively, the Fock exchange part and the correlation part of the self-energy $\Sigma$.

\begin{tabular}{ccccccc}
\hline & $\varepsilon_{n}^{\mathrm{LDA}}$ & $\mu_{\mathrm{x} c, n}^{\mathrm{LDA}}$ & $\Sigma_{\mathrm{x}, n}$ & $\Sigma_{\mathrm{c}, n}$ & $E^{\mathrm{GWA}}$ & $E^{\mathrm{EXP}}$ \\
\hline $4 \mathrm{~s}$ & -6.07 & -9.91 & -12.11 & -1.40 & -9.41 & -9.39 \\
'exact' & -6.06 & -9.93 & -12.36 & & & \\
$4 \mathrm{p}$ & -1.32 & -5.39 & -2.38 & -0.62 & +0.96 & $(0.00)$ \\
'exact' & -1.28 & -5.56 & -2.51 & & & \\
\hline
\end{tabular}

Table 2

The optical absorption peak energy (in eV) of $\mathrm{Zn}$ atom corresponding to the singlet or triplet transition calculated from the BS equation. The experimental value is also listed for comparison.

\begin{tabular}{ccc}
\hline Transition & Present result & Experiment \\
\hline singlet & 6.17 & 5.78 \\
triplet & 3.84 & 4.01 \\
\hline
\end{tabular}

Table 3

Energy gap estimated with the LDA $\left(E_{g}^{\mathrm{LDA}}\right)$ or with the GWA $\left(E_{g}^{\mathrm{GWA}}\right)$ for the pure sodium-chloride crystal and the system with $\mathrm{Cu}$ impurity. The experimental value $\left(E_{g}^{\mathrm{EXP}}\right)$ is shown for the pure crystal.

\begin{tabular}{cccc}
\hline & $E_{g}^{\mathrm{LDA}}$ & $E_{g}^{\mathrm{GWA}}$ & $E_{g}^{\mathrm{EXP}}$ \\
\hline $\mathrm{NaCl}$ & 4.82 & 9.02 & 8.5 \\
$\mathrm{NaCl}: \mathrm{Cu}$ & 2.98 & 8.00 & \\
\hline
\end{tabular}

Next we turn our attention to the sodium-chloride crystal. In Table 2, the band gap estimated from the quasiparticle energies of the top of the valence band and the bottom of the conduction band calculated within the LDA or within the GWA is shown for the pure sodium-chloride crystal and the system with $\mathrm{Cu}$ impurity. The experimental value for the pure crystal is also shown for comparison in this table.

In Fig. 1, the resulting optical absorption spectra of the $\mathrm{Cu}$ impurity system are superposed with those of the pure alkali-chloride crystal, in order to sim- 
ulate the situation of the dilute impurity concentration. The first absorption peak of $\mathrm{Cu}^{+}$ion is located slightly lower in photon energy than that of the pure sodium-chloride crystal. Two (solid and dashed) curves correspond, respectively, the cases of the on-center position and the off-center position of the $\mathrm{Cu}$ atom. Its peak position does not depend on the location of the $\mathrm{Cu}$ ion but its peak height depends. This is due to the transition from $3 d^{10}$ to $3 d^{9} 4 s^{1}$ is the dipole forbidden in the case of the isolated $\mathrm{Cu}^{+}$ion. When the $\mathrm{Cu}$ ion is put in the ligand field, the spherical symmetry is broken and the transition becomes partially allowed. The more deviated the location of the $\mathrm{Cu}$ ion from the on-center position, the stronger this tendency. These resulting characteristics of the absorption spectra are also consistent with the experiment done by Nagasaka $[18,19]$.

\section{$\mathrm{NaCl}: \mathrm{Cu}$ singlet}

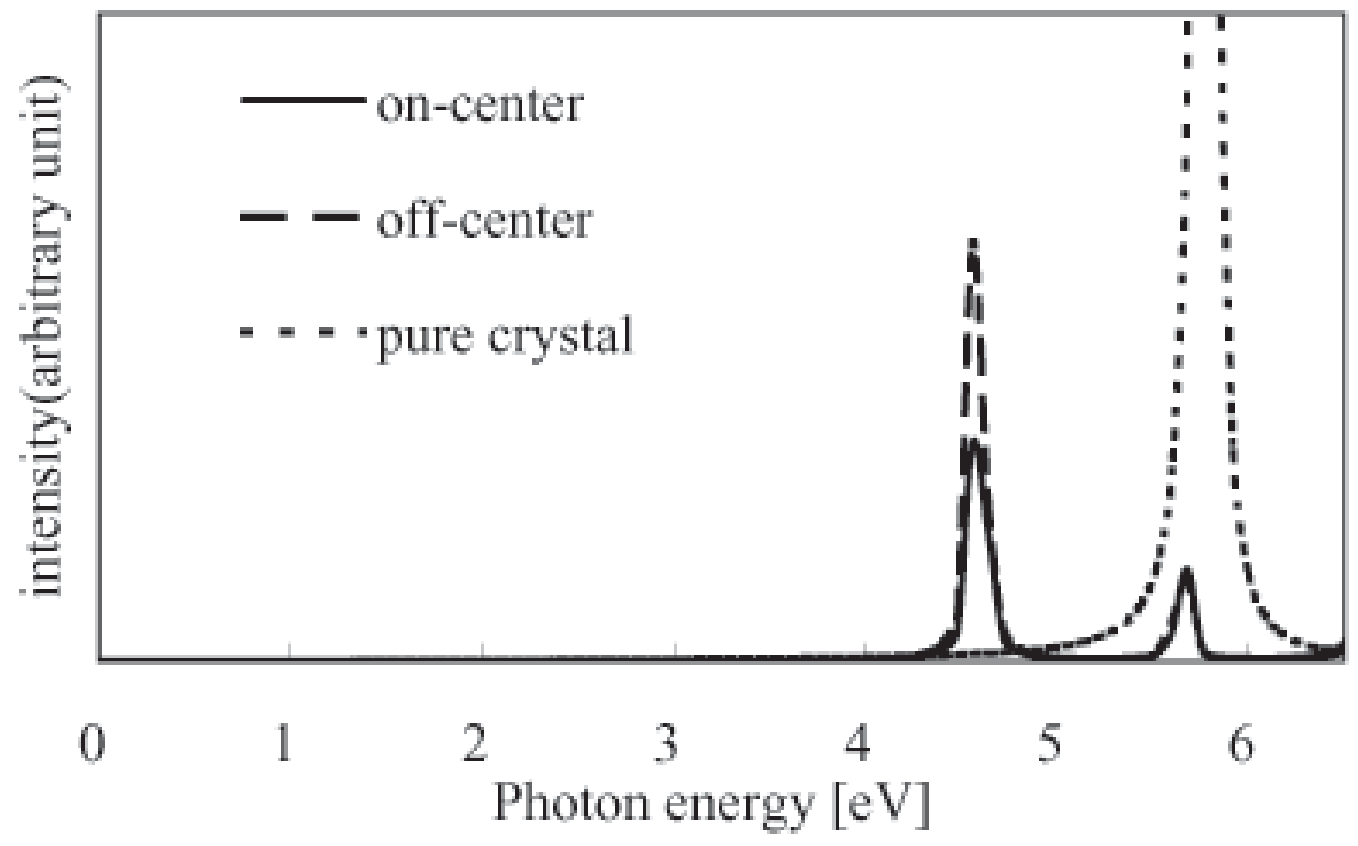

Fig. 1. The calculated photo-absorption spectra of the $\mathrm{NaCl}: \mathrm{Cu}$ system.

\section{Concluding Remarks}

In the paper, we have presented our result of quasipartile energies and optical absorption spectra of an isolated zinc atom and $\mathrm{Cu}^{+}$ions in sodium-chloride crystal. The quasiparticle energies are calculated by the GWA, and the optical absoption spectra are calculated by starting from the GWA and solving the BS equation to take into account the excitonic effect. In the former case, the agreement between our result and the experiment is fairly good, which means the potential ability of the present method. In the latter case, our 
result is consistent with the experiment, i.e., the tendency of the spectrum depending on the position of the $\mathrm{Cu}$ atom is similar to that expected in the experiment. although only $\Gamma$-point calculation is used instead of the $k$-point sampling. In contrast to the conventional quantum chemistry approach such as the configuration interaction (CI) or the multi-reference CI, the present method has an advantage that it can reproduce the spectra of one-particle (quasiparticle) energies and electron-hole (optical absorption) energies all at once without assuming any symmetry of the excited states. The symmetry of the excited states can be seen from the character of the resulting eigenvectors, if needed. The disadvantage of the present method is the computational time which scales as $N^{6}$ when the number of electrons $N$ increases, although the CI calculation is known to scale roughly as $N^{7}$. 


\section{References}

[1] V. Kumar, K. Esfarjani and Y. Kawazoe, Ab Initio Computer Simulations on Microclusters: Structures and Electronic Properties, in: Y. Kawazoe, T. Kondow and K. Ohno (Eds.), Clusters and Nanomaterials: Theory and Experiment, Springer-Verlag, Berlin, Heidelberg, 2002, pp.9-88.

[2] L. Hedin, New Method for Calculating the One-Particle Green's Function with Application to the Electron-Gas Problem, Phys. Rev. 139 (1965) A796-A823.

[3] M. S. Hybertsen and S. G. Louie, Electron correlation in semiconductors and insulators: Band gaps and quasiparticle energies, Phys. Rev. B 36 (1986) 53905413 .

[4] R. W. Godby, M. Schlüter and L. J. Sham, Self-energy operators and exchangecorrelation potentials in semiconductors, Phys. Rev. B 37 (1988) 10159-10175.

[5] S. Albrecht, L. Reining, R. D. Sole and G. Onida, Ab Initio Calculation of Excitonic Effects in the Optical Spectra of Semiconductors, Phys. Rev. Lett. 80 (1998) 4510-4513.

[6] M. Rohlfing and S. G. Louie, Excitonic Effects and the Optical Absorption Spectrum of Hydrogenated Si Clusters, Phys. Rev. Lett. 80 (1998) 3320-3323.

[7] K. Ohno, K. Esfarjani and Y. Kawazoe, Computational Materials Science: From Ab Initio to Monte Carlo Methods, Springer Series on Solid-State Sciences, Vol.129, Springer-Verlag, Berlin, Heidelberg, 1999.

[8] S. Ishii, K.Ohno, Y.Kawazoe and S. G. Louie, Ab initio GW quasiparticle energies of small sodium clusters by an all-electron mixed-basis approach, Phys. Rev. B 63 (2001) 155104;1-6.

[9] S. Ishii, K. Ohno, Y. Kawazoe and S. G. Louie, Ab initio GW quasiparticle calculation of small alkali-metal clusters, Phys. Rev. B 65 (2002) 245109;1-6.

[10] S. Ishii, K. Ohno, V. Kumar and Y. Kawazoe, Breakdown of time-reversal symmetry of photoemission and its inverse in small silicon clusters, Phys. Rev. B 68 (2003) 195412;1-5.

[11] K. Ohno, Optical Properties of Alkali-Earth Atoms and Na2 Calculated by GW and Bethe-Salpeter Equations, Sci. Tech. Adv. Mater. (2004) in print.

[12] K. Ohno, F. Mauri and S. G. Louie, Magnetic Susceptibility of Semiconductors by an All Electron First-Principle Approach, Phys. Rev. B 56 (1997) 1009-1012.

[13] T. Ohtsuki, K. Ohno, K. Shiga, Y. Kawazoe, Y. Maruyama and K. Masumoto, Insertion of $\mathrm{Xe}$ and $\mathrm{Kr}$ Atoms in $\mathrm{C}_{60}, \mathrm{C}_{70}$ Fullerenes and Formation of Dimers, Phys. Rev. Lett. 81 (1998) 967-970.

[14] S. Ishii, K. Ohno and Y. Kawazoe, Dielectric Response Functions Studied by an All-Electron Mixed-Basis Approach, Mater. Trans. JIM 40 (1999) 1209-1212. 
[15] M. Furuya, S. Ishii, Y. Takahashi, S. Nagasaka, T. Yoshinari, Y. Kawazoe and K. Ohno, Stability of Copper Atoms Embedded in Sodium-Chloride Crystals Mat. Trans. 45 (2004) 1450-1451.

[16] G. Strinati, Effects of dynamical screening on resonances at inner-shell thresholds in semiconductors, Phys. Rev. B 29 (1984) 5718-5726.

[17] M. Rohlfing and S. G. Louie, Electron-hole excitations and optical spectra from first principles, Phys. Rev. B 62 (2000) 4927-4944.

[18] S. Nagasaka, Electronic Processes in the Off-Centre Instability for $\mathrm{Cu}^{+}$Impurity Ions in Alkali Chlorides, J. Phys. Soc. Jpn. 50 (1981) 1570-1578.

[19] S. Nagasaka, Off-Centre Instability of $\mathrm{Cu}^{+}$Ions in Alkali Chlorides Due to a Charge-Transfer Excitatoin. J. Phys. Soc. Jpn. 51 (1982) 898-905. 